\title{
Hybrid layer difference between sixth and seventh generation bonding agent
}

\author{
Grace Syavira Suryabrata \\ Department of Conservative Dentistry \\ Faculty of Dentistry Prof. Dr. Moestopo (B) University \\ Jakarta Selatan - Indonesia
}

\begin{abstract}
Since etching is completed at the same stage as priming and bonding, when applying the sixth and seventh generation bonding, the exposed smear layers are constantly surrounded by primer and bonding and cannot collapse. The smear layer and the depth of penetration of resin bonding in dentinal tubules are completely integrated into hybrid layer. The purpose of this laboratory research was to study the penetration depth of two self etching adhesive. Fourteen samples of human extracted teeth were divided into two groups. Each groups consisted of seven samples, each of them was treated with sixth generation bonding agent and the other was treated with seventh generation bonding agent. The results disclosed that the penetration into dentinal tubules of seventh generation bonding agent was deeper than sixth generation bonding agent. Conclusion: bond strength will improve due to the increasing of penetration depth of resin bonding in dentinal tubules.
\end{abstract}

Key words: bonding agent, penetration depth

Correspondence: Grace Syavira Suryabrata, c/o: Bagian Konservasi Gigi, Fakultas Kedokteran Gigi Universitas Prof. Dr. Moestopo (B). Jln. Bintaro Permai Raya No. 3 Jakarta Selatan, Indonesia.

\section{INTRODUCTION}

Dental adhesive system is used to bind bonding resin to email and dentin. Bonding resin substance has developed through generations with changes in chemistries mechanism, packaging/number of bottles, application technique, clinical effectivenes and also in sensitivity technique. ${ }^{1,2}$ Bonding resin is used to cover dentin so that pulp is protected from the movement of oral fluid and contaminating agent. Functionally, bonding resin is regarded as an artificial email. ${ }^{3}$ Several factors influence bonding, among other dentin demineralization, temperature, collagen, dentinal pain, biocompatibility of bonding agent, penetration ability in smear layer to form hybrid layer.

Mineralized dentin causing monomer's inability to diffuse to substrate, therefore dentinal surface has to be etched first. ${ }^{3}$ Etching's function is to form surface texture, so that it can strengthened the bond between restoration agent and bonding resin. ${ }^{4}$ Excessive etching on dentin caused deep demineralization which hampering resin can not completely enter into the collagen fibers at the bottom of demineralized dentin. ${ }^{5}$

Phosphoric acid that remove the smear layer on total etching will cause the collapse of demineralized intertubules dentin matrix if it is dried with air, ${ }^{3}$ whether excessively on sufficiently. ${ }^{6}$ Bonding to dentin with phosphoric acid etching will dramatically increase dentinal permeability causing the raise of flow-out fluid to dentinal surface. Fluid is influential to adhesion because resin has a hydrophobic nature, not adhesive to hydrophilic substrate even after resin tag has been formed inside dentinal tubules. ${ }^{6}$ Bonding agent composition has been developed to form dentinal hybrid and resin tag hybrid. ${ }^{3}$ Bonding to dentin occurs mechanically or chemically. 5

Several researchers stated that chemical bonding gives little influence on bonding dentin resin. It is because bonding dentin resin is generated from a micromechanical reaction result of polymer chain molecules with collagen fibers, called hybrid layer or hybrid zone. ${ }^{3}$ Similar process happened on demineralized dentin, there was no possibility of a chemical reaction between bonding and hydroxi-apatite because there was no calcium or phosphate, it consisted only of collagen fibers and water. ${ }^{6}$

Effort to increase the strength of adhesion is indicated by remove the smear layer with acid, it actually occurs with demineralizing dentin surface under the smear layer. ${ }^{7}$ Nowadays, bonding system without omitting the smear layer but dissolving, is called self-etching adhesives, so that it will make simpler clinical application procedure. Bonding's work method in dentin surface demineralizing process happened at the same time with the penetration of bonding resin according to demineralization depth. ${ }^{8}$ This method happens at the sixth and seventh bonding generations.

Hybrid layer thickness is formed between 2-3 $\mu \mathrm{m}$. Smear layer occurred during email or dentin preparation with the thickness according to the roughness of the bur used. $^{7}$ On hybrid layer with network collagen fiber characteristic, it will form interfiber space almost like the shag carpet on dentin surface, appeared in tubule walls and hybridation of lateral tubules. ${ }^{8}$ 
The sixth generation bonding resin with one stage mechanism composed of two components. Etching depth and bonding resin penetration depth is identical, forming chemical and mechanical bond. Mechanical bond occurs between bonding resin and dentin. Chemically bonding occurs between Calcium hydroxide - apatite and matrix because of phosphoric ester with low $\mathrm{pH}$ as dentin solvent and make an adhesion to dentin. A more simple packaging i.e. two components in one blister is often presenting problem by imperfect mixing causing decreased adhesion strength. Due to the simple application, dentists give less attention to the working manual from the manufacturer. The strength of adhesion will be doubled if applied twice in 15 seconds. ${ }^{5}$

Seventh generation bonding, with one stage mechanism consisted of one component. The process of sixth and seventh bonding generation is similar but the seventh generation has one component, thus avoiding any mistake in mixing. The strength of adhesion to dentin is very good, and three times application subsequently in 30 seconds has enabled deeper resin penetration into dentinal tubules. ${ }^{9}$

Bonding resin bond to dentin depends on the penetration of bonding resin into the smear layer and on the depth of bonding resin penetration into the dentinal tubules. The deeper the penetration of bonding resin into dentinal tubules, the better bonding resin retention to dentin will be.

The purpose of this research was to identify the different of penetration ability from the of sixth and seventh bonding generations into dentinal tubules. The benefit of this study is to fully understand the penetration ability of sixth and seventh generation bonding resin into dentinal tubules.

\section{MATERIALS AND METHODS}

Materials used in this research was 14 caries-free and post-extraction of left or right upper jaw first premolar teeth, , two bonding agent types: sixth generation (Adper Prompt L-Pop made by 3M ESPE USA) and seventh generation (iBond made by Heraeus Kulzer GmbH and Co KG Germany), and acrylic self curing platinum. The tools used in this study was metal diamond disc typed 7016 (Carlo Italy), scanning electron microscope (SEM) made in UK, platinum coater tool (Polaron Sputter Coater), rough polisher machine, smooth polisher machine (Buehler Polisher Ecomet III Grinder), light curing instrument (Litex USA), bonding brusher, micrometer, vacuum set, and dental pinset.

Extracted teeth were rinsed with water, dried with gauzed cloth, occlusal surface was cleaned with metal diamond disc around $3 \mathrm{~mm}$ from central fissure. The surface was cut, dampened with flowing water and blowed gently with low pressure air (less than 2 atm) until the dental surface was wet and shiny. Afterwards, bonding agent was applicated with new brush for every sample following manual instruction of each substance. Seventh generation bonding agent needed 20 seconds light curing polimerization and sixth generation needed 10 seconds. Then, the tooth was cut at bucco palatal side, and cut again to mesio distal direction. The tooth was rinsed with aquades and dried. Two casts, 1 inch diameter was pasted at its bottom with adhesive laque band, until closely tied. The tooth was removed with pinset and the exposed surface was pasted to the base of the dental cast. Acrylic self curing was inserted to the dental cast until the tooth was soaked with $9 \mathrm{~mm}$ thickness. Moreover, a small label was drowned

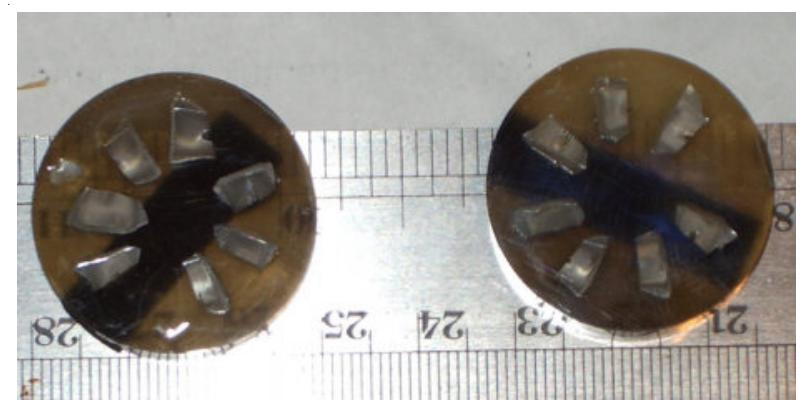

Figure 1. Dental samples implanted in acrylic, coated with platinum and vacuumed.

inside liquefied resin to unite with resin and to be read easily from sample side. After 1 hour, resin hardened and dental cast was opened. Acrylic was then polished until it reached $7 \mathrm{~mm}$ thickness, the exposed surface was again polished until smooth using alumina and nylon cloth. The sample was coated with platinum using Platinum coater and in the end was vacuumed (Figure 1).

The samples were observed with scanning electron microscope (SEM), with 5.90 kilo electron volt resolution. After hybrid layer and the length of bonding resin penetration into dentinal tubules were seen, it was photographed, the result was printed, magnified by 3000 times. The length of bonding resin tag to dentinal tubules was measured with micrometer directly from SEM monitor screen. SEM examination was intended to observed the penetration depth of bonding resin agent into dentinal tubules from dentinal surface.

\section{RESULTS}

The measurement of bonding resin penetration depth in dentinal tubules on 2 treatment groups has shown in table 1 . 
Table 1. Average value and standard deviation of bonding resin penetration into dentinal tubules $(\mu \mathrm{m})$

\begin{tabular}{cccc}
\hline Group & $\begin{array}{c}\text { Sample } \\
\text { Number }\end{array}$ & Mean & $\begin{array}{c}\text { Standard } \\
\text { Deviation }\end{array}$ \\
\hline 1 & 7 & 12.451 & 2.51 \\
2 & 7 & 9.471 & 2.07 \\
\hline
\end{tabular}

Note:

Group 1: Applicated with seventh bonding generation

Group 2: Applicated with sixth bonding generation

To identify the different result of treated groups, statistical Independent T Test $(p=0.05)$ was utilized. The result showed that $\mathrm{T}$ Test observed probability was 0.032 meaning there was a significant difference between 2 groups, first group with sixth generation bonding resin (Adper Prompt L-Pop) and second group with seventh generation bonding resin (iBond). SEM examination demonstrated the depth of seventh generation bonding resin agent was longer than the sixth generation (Figure 2 and 3 ).

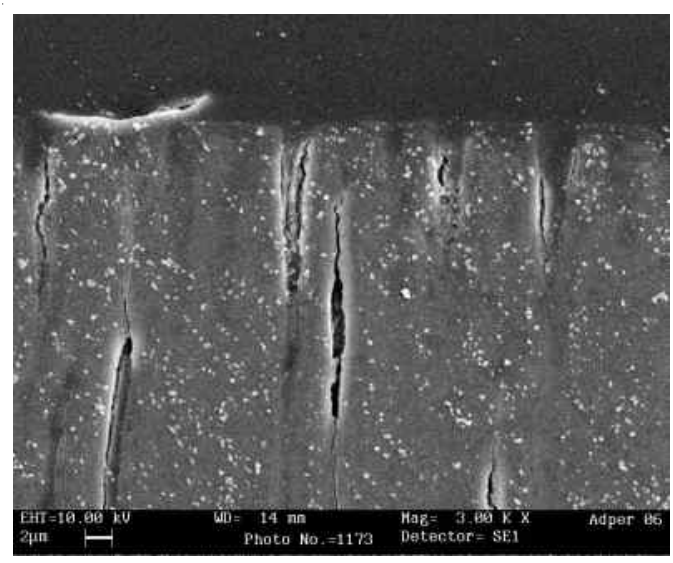

Figure 2. Sixth generation bonding penetration length (SEM picture).

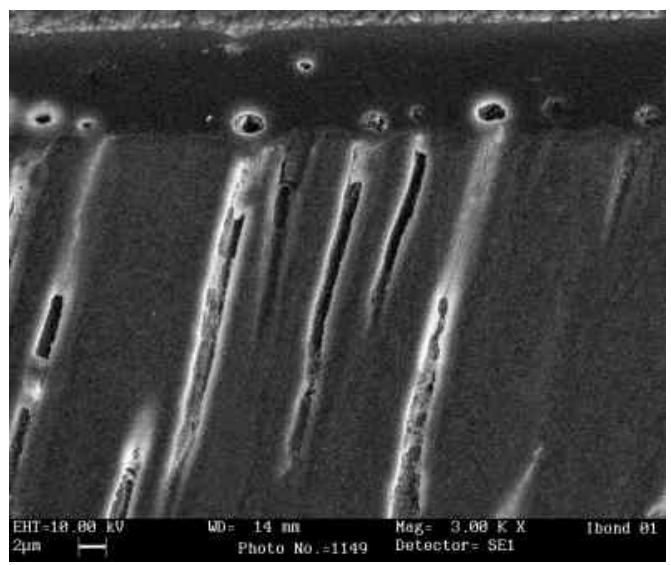

Figure 3. Seventh generation bonding penetration length (SEM picture).

\section{DISCUSSION}

The adhesive mechanism of both bonding agent groups was to maintain the smear layer, dissolved and exploited as hybrid layer formed between dentin and resin; not resin and neither dentin, but hybrid from both substances. Bonding will cover collagen fibers and move on penetrating into dentinal tubules to form resin tag. ${ }^{1}$

The different packaging and application technique of both groups i.e.: seventh generation bonding was packed in one bottle without premixed while sixth generation bonding was packed in two components subsequently needed premixing. Seventh generation bonding was apply to dentin for three times in 30 second, whereas the sixth generation bonding was treated once in 15 seconds. ${ }^{10,11}$

The benefit of self-etching adhesive which was a combination of etching, primer and adhesive in one stage, was in lessening work time and mistakes of any work stage. ${ }^{12}$ The bond of two bonding groups occurred through monomer as the result of released protons by phosphoric acid 4 META, estherized by HEMA. At the time of etching process, methacrylate polymerized with $\mathrm{Ca}^{++}, \mathrm{pH}$ inclined slowly, causing decreased acidity and etching process will stop. ${ }^{11}$ The depth of etching was similar with resin penetration depth. ${ }^{10}$ The length of bonding resin penetration into dentinal tubules was influenced by the length of etching acid reaction from acid $\mathrm{pH}$ until it became normal $\mathrm{pH}$ inside dentinal tubules. The longer the reaction time, the deeper the bonding resin penetration into dentinal tubules. The deeper the penetration, the better bonding resin retention to dentin. Seventh bonding generation needed 30 seconds to etching acid reaction as mentioned in the manufacturer's manual, while the sixth generation needed only 15 seconds.

The result of Independent $T$ Test was a significant difference of bonding resin penetration depth between seventh and sixth generation $(\mathrm{p}<0.05)$. The average value of the first group bonding penetration depth (seventh generation) was $12.451 \mu \mathrm{m}$ and the second group (sixth generation) was $9.471 \mu \mathrm{m}$. Therefore, based on the average penetration depth value, seventh bonding generation was deeper than sixth generation, or tag resin from seventh generation was longer than the sixth generation. It was illustrated as follows (Figure 4).

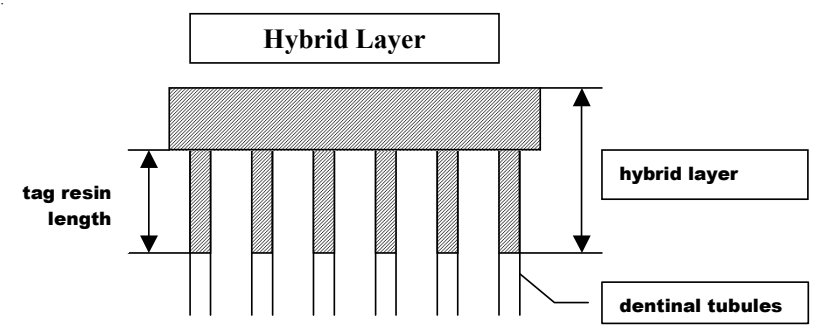

Figure 4. Hybrid layer of dentinal surface with bonding-dentin resin inter surface in the form of hybridizing plaited collagen fibers and hybridizing in dentinal tubules. 
From Independent $\mathrm{T}$ Test, the average length of tag resin of the second group was longer than the first group. The longer tag resin of hybrid layer will produce better hybrid layer retention. It can be concluded that the seventh generation bonding resin hybrid layer has better retention into dentin compared to the sixth generation bonding resin.

\section{REFERENCES}

1. Dunn JR. iBond: The seventh - generation, one - bottle dental bonding agent. J Com $2003 \mathrm{Feb}$; 24(2):14-18.

2. Bowen RL, Harjenhoff WA. Development of an adhesive bonding system. Operative Dentistry 1992; 5(Supplement):75-80.

3. Nakabayashi N, Pasley DH. Hibridization of dental hard tissues. Tokyo, Chicago, Berlin, London, Paris, Barcelona, Sao Paulo, Moscow, Prague and Warsaw: Quintessence Publ Co Ltd 1998; p. 14-17, 37, 92-93.

4. Allbeury J. New development prompt a rethink on self etching adhesives. Special Report Dental Practice March-April 2004; $114-9$.
5. Baier RE. Principles of adhesion. Operative Dentistry 1992; 5(Supplement):1-9.

6. Roberson TM, Heymann HO, Swift EJ. Sturdevant's art and science of operative dentistry. $4^{\text {th }}$ ed. St Louis, London, Philadelphia, Sydney, Toronto: CV Mosby Co; 2002. p. 177-85.

7. Oliviera SSA, Pugach MK, Hilton JF, Watanabe LG, Marshall SJ, Marshall Jr GW. The Influence of the Dentin Smear Layer on Adhesion: Self-Etching Primer VS total etch system. Dental Materials 2003; 19(8):758-67.

8. Summits JB. Fundamental of operative dentistry. $2^{\text {nd }}$ ed. Chicago, Berlin, Tokyo, Paris, London, Sao Paulo, Barcelona, Moscow, Prague and Warsaw: Quintessence Pub Co Inc; 2001. p. 196-8, 208-11.

9. Pitel M. Bonding general conditions. Quintessence Publ Co Inc. Office Online Publ. Ed. J Adhes Dent 2003; 20(8):1-22.

10. iBond Info. Available at: www.ibond-info.com. Accessed November 28, 2004.

11. Technical Product Profile. Adper Prompt L-Pop and Adper Prompt. Special Report 3 M Espe. 2004; 1-21.

12. Tay FR, Pashley DH. Aggressiveness of contemporary self etching systems. I: Depth of penetration beyond dentin smear layers. J Den Mat 2001 August; 17:296-308. 